\title{
A Hysteresis Dynamic Mathematical Model Approach to Parametric Estimation System
}

\author{
Nubia Ilia Ponce de León Puig ${ }^{D}$, Leonardo Acho $\mathbb{D}^{D}$, and José Rodellar \\ Department of Mathematics, Universitat Politècnica de Catalunya, Barcelona, Spain \\ Correspondence should be addressed to Nubia Ilia Ponce de León Puig; nubiliaponcepuig@gmail.com
}

Received 11 November 2020; Revised 10 January 2021; Accepted 2 February 2021; Published 12 February 2021

Academic Editor: Francesco Tornabene

Copyright ( $\odot 2021$ Nubia Ilia Ponce de León Puig et al. This is an open access article distributed under the Creative Commons Attribution License, which permits unrestricted use, distribution, and reproduction in any medium, provided the original work is properly cited.

\begin{abstract}
The main contribution of this paper is the proposal of a recent hysteresis dynamic model which is successfully employed within a posited signal modulator. The modulation of signals is a commonly required stage in many engineering applications, such as telecommunications, power electronics, and control, among others. In this paper, the effectiveness of a signal modulator based on the well-known Delta modulator when it contains a dynamic hysteresis system within its main structure is presented. To do that, it is resorted to an application of the granted Hysteresis-Delta Modulator. This application consists of including the modulator within an adaptive scheme, since it is well known that the persistent excitation condition is required, for instance, in parameter estimation tasks. Hence, the main functional property of the modulator with hysteresis is its ability of producing a modulated signal with uniform high-frequency content even when its input is not a permanent persistent excitation signal. To highlight the main contribution of this paper, a numerical experiment of a parameter estimation system is developed to compare the performance of the modulator with the proposed hysteresis model and two other previously reported hysteresis systems. That is, three different scenarios have been tested in the parameter estimation of a nonminimum phase system. Finally, the numerical experiments confirm that the proposed hysteresis model along with the modulator provides the best performance as expected.
\end{abstract}

\section{Introduction}

In recent years, the comprehension of the hysteresis phenomenon and the development of adequate mathematical tools to describe it have attracted the attention of many researchers [1-6]. The main reason is that hysteresis is encountered in many different physical and mathematical fields. Examples include magnetic, mechanical, and optical systems [1, 7-9]. It is also well known that the phenomenon of hysteresis is a complex physical process that represents an important challenge, for instance, to model and control systems that contain this effect. Moreover, hysteresis is, at the same time, a source of strong technological progress for some crucial scientific developments $[3,10]$.

In some engineering applications, hysteresis can be described as a nonlinear phenomenon that, under zero-bias and low-frequency periodic excitation, its output has a periodic response with the same frequency of its input $[4,6]$.
Usually, hysteresis systems have an output versus input response that plots a historical closed-loop trajectory. Hence, it makes that hysteresis be linked to the formation of loops, and these loops may take a variety of different forms depending on its input excitation and the parameters that govern the hysteretic system [1-3]. Besides, hysteresis can be described as a dependence of the state of a system on its time history $[1,5]$. Therefore, hysteresis also provides a memory effect.

Mathematical modelling of hysteresis is an important challenge due to the hysteresis effects that are invoked in a variety of systems; such as mechanical, ferromagnetic, or ferroelectric systems, to name a few [6]. Recently, the necessity to control this kind of systems has grown $[4,11]$. Moreover, it is well known that some modern control algorithms require the knowledge of a mathematical model of the plant to be controlled, thus an adequate hysteresis model is also necessary to control systems that contain this 
nonlinear phenomenon. Equally, an adequate model of hysteresis may be useful to improve the performance of controllers by introducing its behaviour in the control scheme [12]. This idea may solve the usual control problems due to saturation, parameter drift, and bursting effect $[12,13]$. Additionally, hysteresis models can be used in the design and characterization of novel actuators [14]. In general, there are two options to model hysteresis. One alternative is to create complex models that almost accurately reproduce the hysteresis phenomenon. However, these kinds of models are too arduous to be used in practical applications. Hence, the common alternative is to develop simple hysteresis models which, although not giving the best description of the physical hysteresis behaviour of the system, do keep relevant input-output features useful for characterization, design, and control purposes $[4,5,15]$.

Through the years, different mathematical models of hysteresis have been developed. For instance, in some magnetic applications, hysteresis is the main property used to produce permanent magnets and to manufacture magnetic recordings and energy conversion devices [2, 3]. Likewise, hysteresis considerably improves the performance of vibration control algorithms in the civil engineering structure [4]. In these applications, the hysteretic Bouc-Wen model is traditionally employed in the control design stage $[7,16,17]$. On the contrary, the hysteretic Preisach model is useful to design magnetic and piezoceramic actuators $[14,18]$. In addition, other hysteresis models are also utilized in the area of actuator design $[19,20]$. Supplementary to this, hysteresis has been invoked to avoid saturation effects [13]. Finally, modulators also use hysteresis to adapt signals according to their application objective $[12,21,22]$. However, to the best knowledge of the authors, these modulators have never been used to improve parameter estimation processes as it is done in this paper.

Motivated by the above statements, in this paper, a new hysteresis dynamic model is conceived, which is expected to be applied in some engineering applications because of its special characteristics. Nevertheless, here, the hysteresis system model is used to redesign a signal Delta Modulator. Furthermore, the modulator will be applied to a parametric estimation process to improve its performance. To highlight the potential of the contribution, two previous cited hysteresis models are used within the modulator, as well as the model proposed here. Afterwards, a comparative study is done with the results obtained by using the three different models above mentioned. The first hysteresis case consists of a relay based on a static system provided by Simulink, Matlab [12], the second one is a hysteresis dynamic model proposed in [5]; and the third case is the one with the mathematical hysteresis model. Finally, the modulator scheme with these three different cases will be employed in the parametric estimation process of a nonminimum phase system.

The signal modulation method carried out here follows the main idea of the standard one-bit-analog-to-digital Delta modulator converter [23]. This kind of modulator has had a great variety of applications in digital communication transmission [23], secure communication design [24, 25], and some patents related to sinusoidal signal generators [26] and sensors for measurements [27]. As mentioned before, this proposed hysteresis model is integrated into a Delta modulator structure and then applied to a parameter estimation process. Therefore, according to our numerical experiments, this development increases the Delta modulator performance in this specific application.

In the field of adaptive control systems, parameter estimation is mandatory when the plant or process to be controlled has parameters that are continuously changing or when the process is too complex and the basic physical processes' model is not fully understood [28]. Thus, parametric estimation is applied to obtain the information of the plant, more specifically, to obtain information of the plant parameters that allow the control law to be automatically adjusted and then achieving its control objective. On the contrary, adaptive control based on linear parameter estimation is notably effective when this parameter estimation process is persistent excited [28]. Therefore, it results are important to develop a system that is able to supply this persistent excitation condition required by the parameter estimation process [29]. Hence, it is shown that the Hysteresis Delta Modulator (HDM) proposed in [12], and here carefully redesigned, can provide the above-mentioned features. What is more, it is expected that the Hysteresis Delta Modulator with our proposed hysteresis model supplies a better rich persistent excitation signal in comparison with two other basic hysteresis models.

Summarizing, the main contributions of this paper are as follows:

(1) To propose a recent hysteresis dynamic model that may be applied in different engineering systems

(2) To redesign a Hysteresis Delta Modulator that is able to improve the performance of a parameter estimation algorithm

\section{The Hysteresis Delta Modulation Scheme}

This section introduces the concept of Delta Modulator with hysteresis. This scheme is motivated by a digital Delta Modulator, which is a 1 bit analog-to-digital converter. This converter consists of transforming a continuous time signal into a train of pulse data streams [12, 23, 24]. This modulator is essentially used, for instance, in the transmission of digital voice information [23].

The structure of the Delta modulator consists of a comparator or quantizer in the forward path and an integrator in the feedback path of a given simple control loop [24]. The aim of the quantizer is to convert the difference between the input signal and the average of the previous steps. That is, a comparator that may give one of two possible values depending on the input signal. In this paper, the arrangement called the Hysteretic Delta Modulator (HDM) proposed in [12] replaces the comparator by a hysteresis model. Then, it is expected that the dynamic hysteresis system performs the same task of the quantizer in its basic principle but with the advantage of having a dynamic 
behaviour. This outcome scheme is shown in Figure 1. In this approach, the modulated signal is a train of pulses that takes two values. Furthermore, it is important to highlight that the information of the input signal can be easily retrieved by using a low-pass filter at the modulator output [30]. By the way of illustration, Figure 2 shows a sinusoidal input to the HDM, and its modulated signal output is obtained as a train of pulse signals. Finally, to retrieve the original signal, a lowpass filter is required. Later on, it will be shown that the modulated signal is one with high-frequency content, which means it is a persistent excitation signal. Here, this modulator is amenable to improve the parameter estimation performance.

To expose how the Hysteresis Delta Modulator works along with the parameter estimation, Figure 3 shows the parameter estimation block diagram with the HDM scheme. It is useful to observe that the input to the Hysteresis Delta Modulator block may correspond to a time-variable set point or a given control signal. Furthermore, the parameter estimation block in Figure 3 uses this modulated signal which is a persistent excitation data to appropriately activate the estimation system dynamics. On the contrary, and hereinafter, it is assumed that the process to be employed has a low-pass filter property, meaning that it is able to naturally retrieve the information coded in the modulated signal. This property is commonly assumed in the adaptive control design [28].

\section{Mathematical Hysteresis Model Description}

The main objective of this section is to introduce the recent hysteresis model. Besides, two hysteresis systems are recalled for comparison purposes. This section begins by invoking these models, labelled here as Case 1: Hysteresis by Using Static Relay and Case 2: Hysteresis Dynamic Model. After that, our proposed model is exposed, labelled as Case 3: The Proposed Hysteresis Dynamic Model. Finally, numerical experiments are well implemented to highlight the main dynamics' characteristics of each model.

3.1. Case 1: Hysteresis by Using Static Relay. This case is the well-known hysteretic relay system based on two delayed signum functions. This model is provided, for instance, by Simulink of Matlab and named as the Relay block [12]. This Simulink block is programmed by selecting the values for the switch on/off transition time and the output on/off parameter values. Figure 4 gives a system response example generated by using the values of switch on/off $=\{-0.1,0.1\}$ and output $o n / o f f=\{-2,2\}$, and the input is set as a sinusoidal signal with amplitude 4 and frequency $0.05 \mathrm{~Hz}$.

3.2. Case 2: Hysteresis Dynamic Model. This particular model was originally proposed for the chaotic generator design [15] and later used, for instance, to avoid saturation in controlled wind turbines [13]. This system is stated as follows:

$$
\dot{z}(t)=\alpha[-z(t)+b \operatorname{sgn}(x(t)+a \operatorname{sgn}(z(t)))],
$$

where $a$ and $b \in \mathfrak{R}^{+}$are the hysteresis loop parameters and $z(t)$ is the internal variable of the model (see Figure 5 ). The transition time rate between $b$ and $-b$ is governed by the real positive parameter, $\alpha$; $\operatorname{sgn}(\cdot)$ is the signum function, and $x(t)$ is the input signal.

3.3. Case 3: The Proposed Hysteresis Dynamic Model. In this section, the proposed hysteresis model is presented. As in the previous case, the original idea of our model arises from the electronic point of view, where its main theoretical foundation consists of employing the signum function as a representation of a memory action given by electronic relays [5]. Besides, this model adequately manipulates the input signal to conform the desired hysteretic dynamic behaviour by employing the equivalent information related to position and velocity. This model is as follows:

$$
\begin{aligned}
\dot{w}(t)= & -\alpha[w(t)-b \operatorname{sgn}[x(t) \\
& -\operatorname{asgn}(\dot{x}(t)) \operatorname{sgn}(x(t))+\operatorname{asgn}(\dot{x}(t))]],
\end{aligned}
$$

where $a$ and $b \in \mathfrak{R}^{+}$are the parameters of the hysteresis loop, $\alpha \in \mathfrak{R}^{+}$is the transition time rate (see Figure 5) and $w(t)$ is the internal variable. Finally, $\operatorname{sgn}(\cdot)$ is the signum function, and $x(t)$ is its input signal.

3.4. Particularities of the Hysteresis Models. The properties of the models for Cases 2 and 3 are illustrated in the following numerical simulations. Case 1 is omitted since it is well represented in Figure 4. These experiments consist of analysing the hysteresis response of the above-cited models under two different input signals. The first input is depicted in Figure 6(a) and is given by the following:

$$
x_{1}(t)=6 \sin (2 \pi t) e^{-0.1 t}
$$

and the second input (see Figure 6(b)), is $x_{2}(t)=-x_{1}(t)$. The hysteresis parameter values used for numerical experiments are set in both cases as $a=1, b=5$, and $\alpha=50$.

The results obtained from the model presented for Case 2 are shown in Figure 7. Here, it is possible to observe a difference on the hysteresis direction between graphics shown in Figure 7(a) by using input $x_{1}(t)$ and in Figure 7(b) by employing input $x_{2}(t)$. Moreover, it is observed that both hysteresis responses are centred with respect to the graphic origin point.

Likewise, Figure 8 shows the simulations for our model (2) by using the same scenario than the previous case, that is, the same hysteresis parameter data and the same test input signals. From this figure, the shape of the hysteresis loop changes its position and direction with respect to the graphical origin point according to the employed input as it is shown in Figures 8(a) and 8(b), respectively.

Remark 1. Given models (1) and (2), it is possible to convexly linearly combine both response systems into one as follows:

$$
\omega(t)=\alpha_{0} z(t)+\left(1-\alpha_{0}\right) w(t) ; \quad 0 \leq \alpha_{0} \leq 1 .
$$




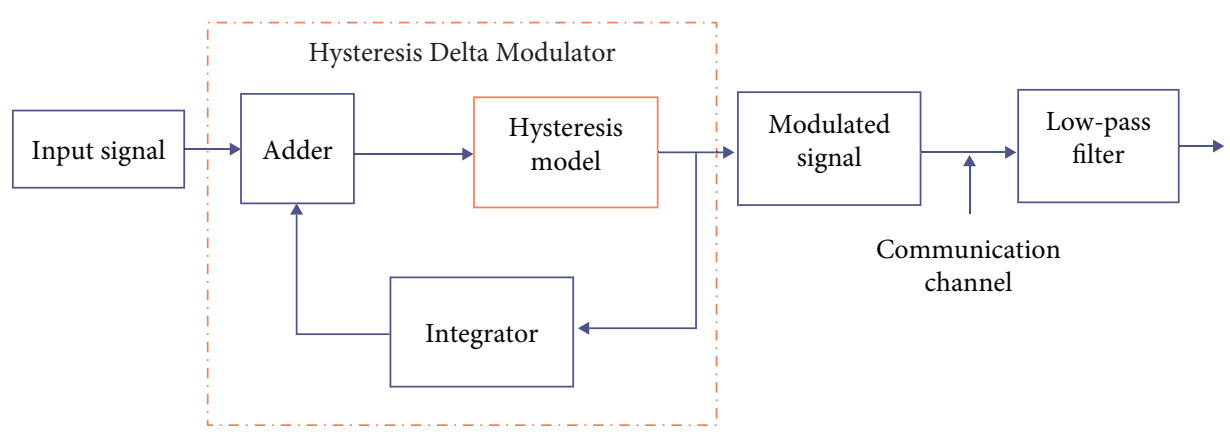

Figure 1: The Hysteretic Delta Modulator scheme.

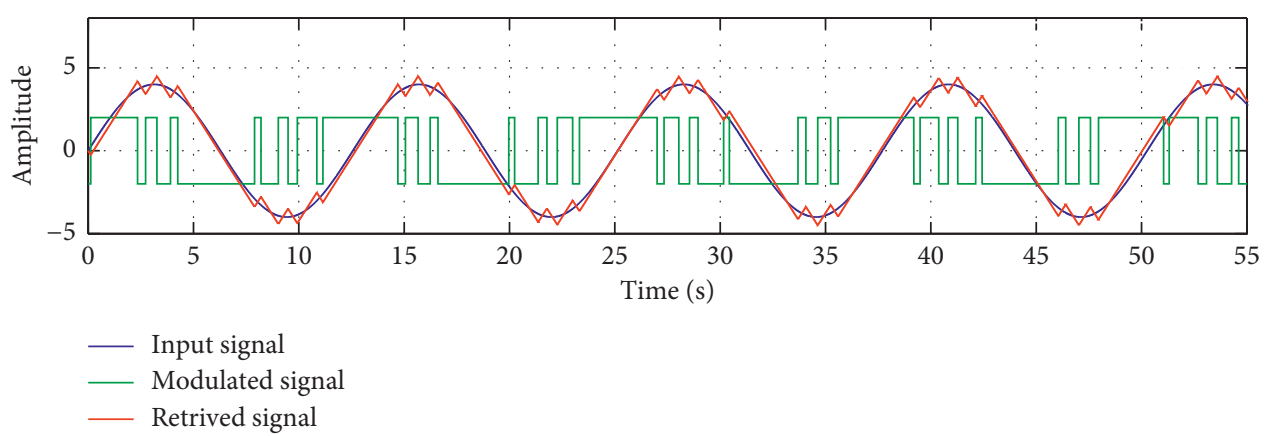

Figure 2: Example of the Hysteretic Delta Modulator operation.

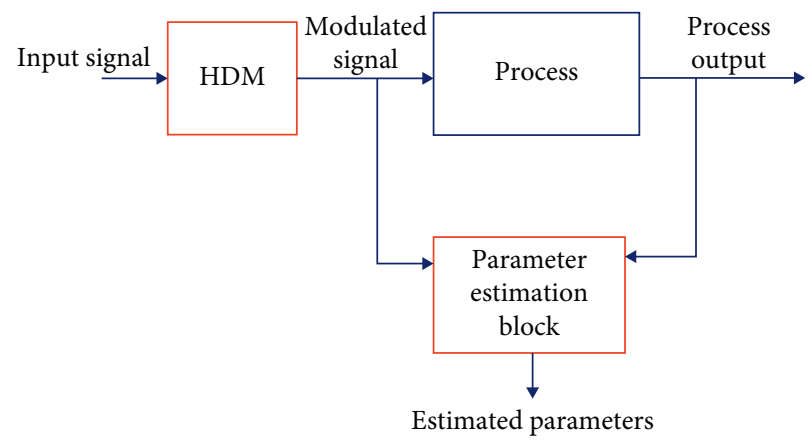

FIgURe 3: Parametric estimation block diagram for the HDM scheme.

An example of the hysteresis loop obtained with the equation in (4) is depicted in Figure 9 when $x(t)=x_{1}(t)$ and $\alpha=0.5$.

\section{Parameter Estimation of a Nonminimum Phase System}

This section introduces a well-known parameter estimation algorithm applied to a nonminimum phase system. This will be further employed in the next section in our numerical experiment realization. The proposed nonminimum phase system is [31]:

$$
G(s)=\frac{a_{1} s+a_{2}}{s^{2}+b_{1} s+b_{2}}=\frac{s-2.28}{s^{2}+0.57+5.14} .
$$

Recalling that nonminimum phase systems are characterized because these models have an unstable inverse [32]. This unstable inverse characteristic induces, for instance, a notable challenge for the adaptive control design [33]. On the contrary, system (5) is employed here due to the wide spectrum on its parameter values: $\left\{a_{1}, a_{2}, b_{1}, b_{2}\right\}=\{1,-2.28,0.57,5.14\}$.

To implement the parametric estimation system, the time-domain Gradient Algorithm technique is invoked [28]. This technique parts from the time-domain expression of the process and requires signals that may be obtained by filtering its input and output as it is shown in Figure $10[28,34]$.

To begin with the parameter estimation algorithm design, the process in (5) is first expressed and parameterized in the time-domain framework as follows:

$$
\ddot{y}(t)+b_{1} \dot{y}(t)+b_{2} y(t)=a_{1} \dot{u}-a_{2} u,
$$

where $a_{1}, a_{2}, b_{1}$, and $b_{2}$ are the system parameters to be estimated and assumed unknown for the parameter estimation block. Previously, it was mentioned that the input 


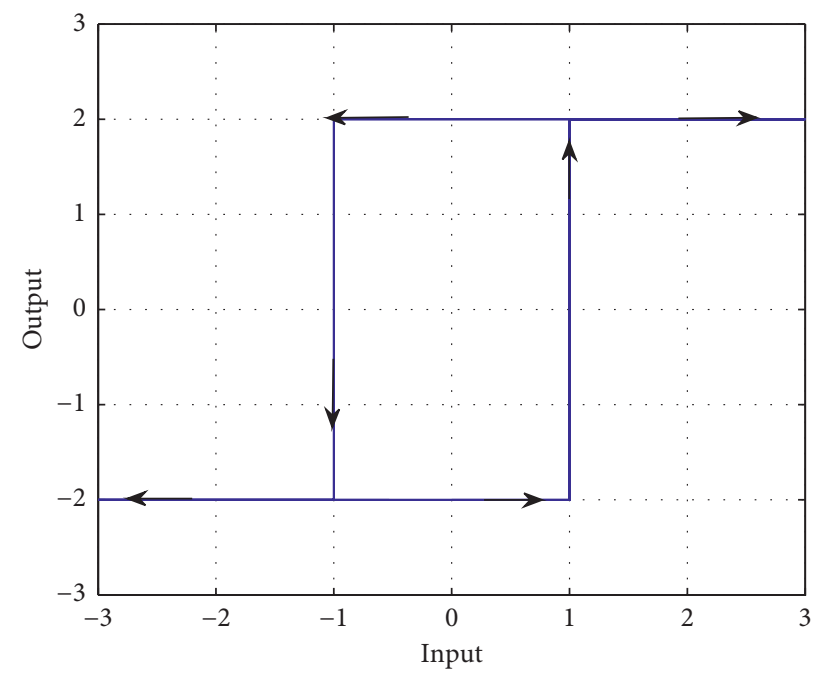

FIGURE 4: Input versus output response of the hysteresis relay block.

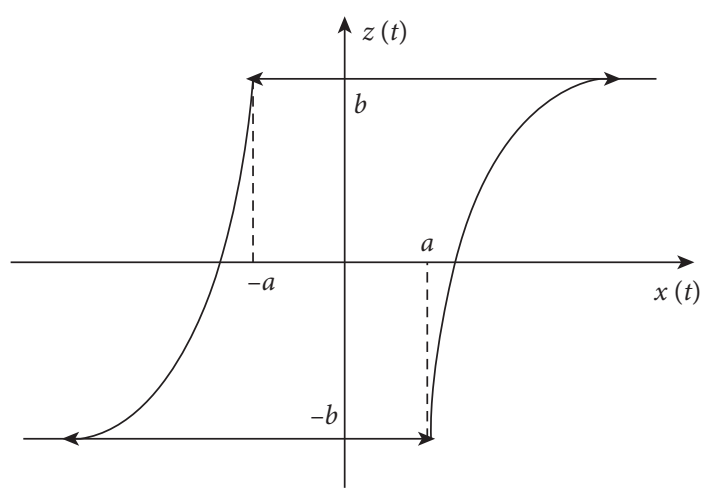

Figure 5: Hysteresis behaviour $z(t)$ versus $x(t)$ of system (1).

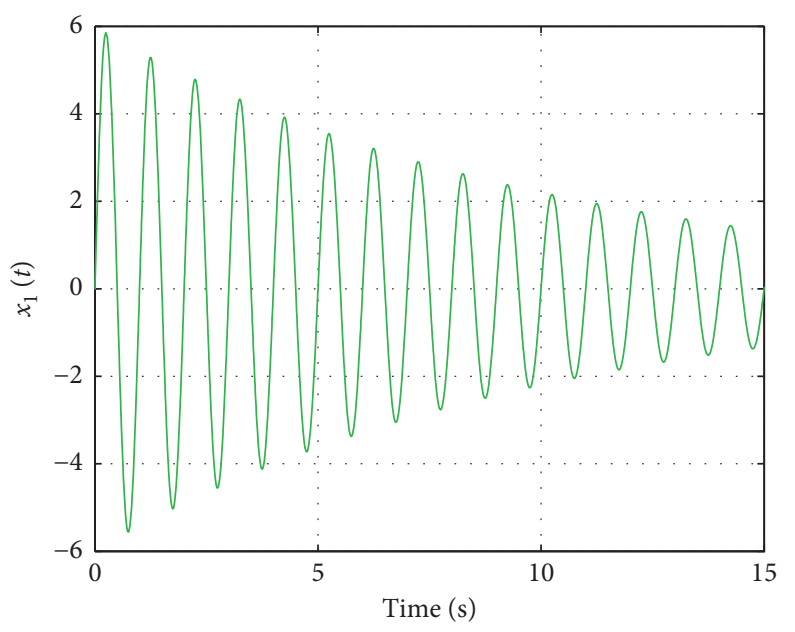

(a)

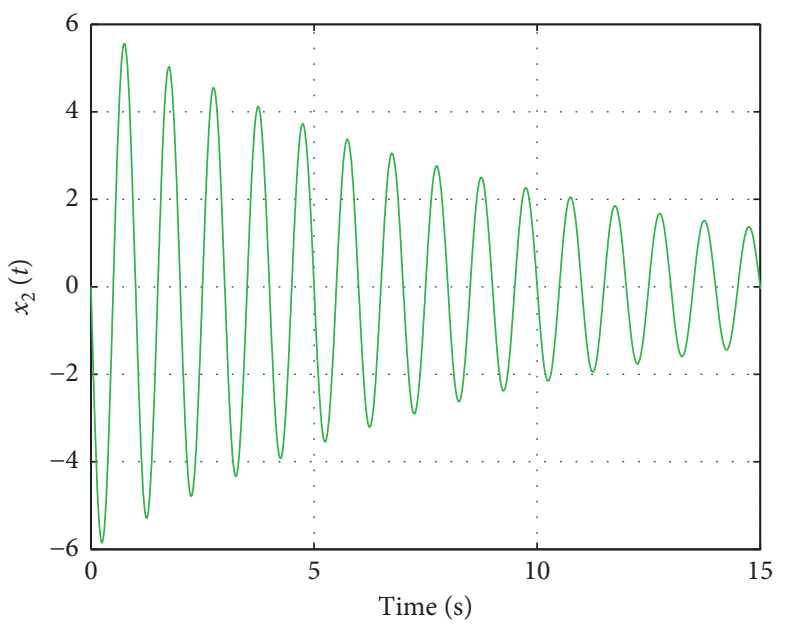

(b)

FIgURE 6: Test input signals. 


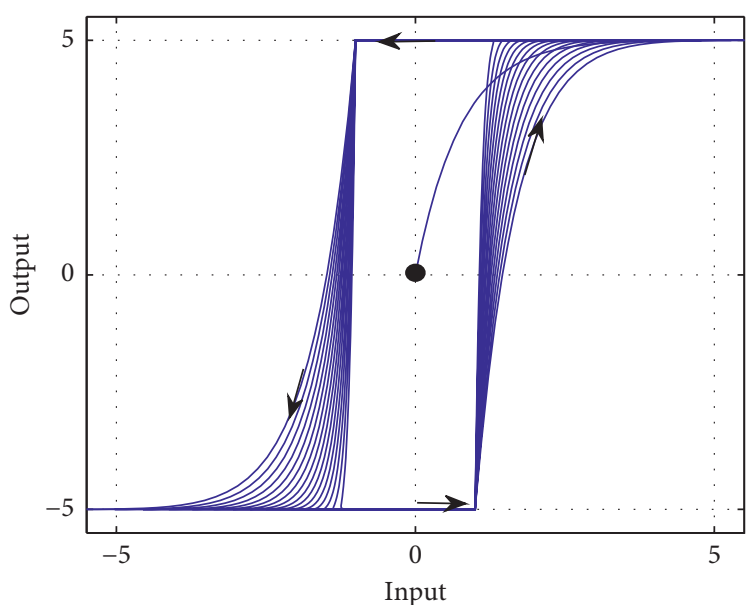

(a)

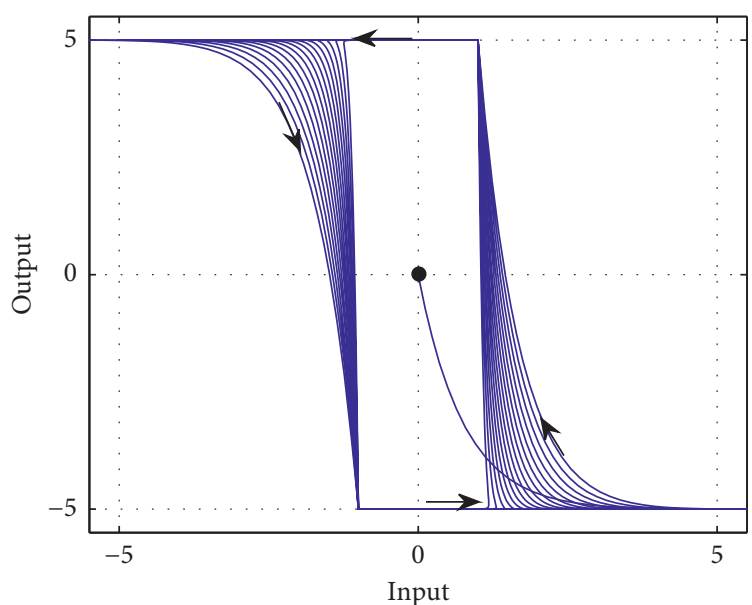

(b)

FIgURE 7: Hysteresis behaviour of system (1).

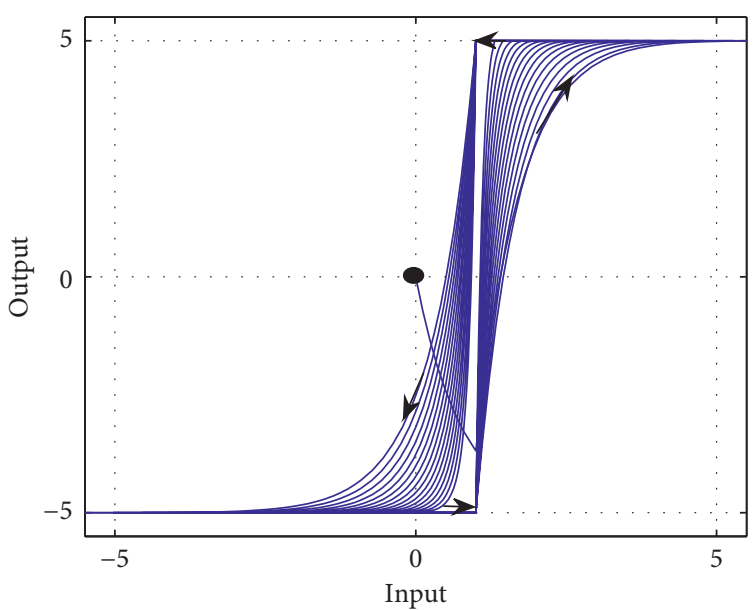

(a)

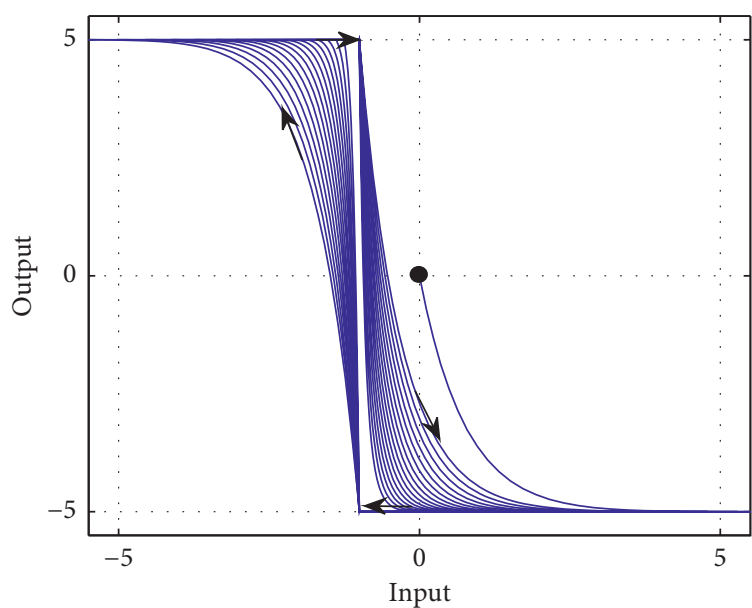

(b)

FIgURE 8: Hysteresis behaviour of system (2).

and output signals are filtered; thus, two second-order stable filters are selected as follows:

$$
\begin{aligned}
& G_{F_{1}}(s)=\frac{s}{s^{2}+\lambda_{1} s+\lambda_{2}}, \\
& G_{F_{2}}(s)=\frac{1}{s^{2}+\lambda_{1} s+\lambda_{2}},
\end{aligned}
$$

where $\lambda_{1}$ and $\lambda_{2}$ are real positive gains properly chosen by the designer. Then, the process output can be stated as follows:

$$
Y(s)=\left[a_{1}, a_{2}, B_{1}, B_{2}\right]\left(\begin{array}{c}
u_{f_{2}} \\
u_{f_{1}} \\
y_{f_{2}} \\
y_{f_{1}}
\end{array}\right)=\theta^{* T} \varphi,
$$

where $\theta^{* T}$ is the parameters' vector and $\varphi$ is the regression matrix formed by the filtered signals: $u_{f_{2}}$ and $u_{f_{1}}$ for the input plant and $y_{f_{2}}$ and $y_{f_{1}}$ for the output plant. Besides, $B_{1}=\lambda_{1}-b_{1}$, and $B_{2}=\lambda_{2}-b_{2}$. In the time domain, from system (8), the following expression is obtained:

$$
\hat{y}(t)=\left[\widehat{a}_{1}, \widehat{a}_{2}, \widehat{B}_{1}, \widehat{B}_{2}\right]\left(\begin{array}{l}
u_{f_{2}} \\
u_{f_{1}} \\
y_{f_{2}} \\
y_{f_{1}}
\end{array}\right)=\widehat{\theta}^{* T}(t) \varphi(t),
$$

where $\widehat{a}_{1}, \widehat{a}_{2}, \widehat{B}_{1}$, and $\widehat{B}_{2}$ are the estimated signals to $a_{1}, a_{2}, B_{1}$, and $B_{2}$, respectively. Finally, by following the Gradient Algorithm method, the time-domain parameter update law is obtained [28]:

$$
\dot{\hat{\theta}}=-\gamma \varphi(t) e(t), \quad \gamma>0
$$




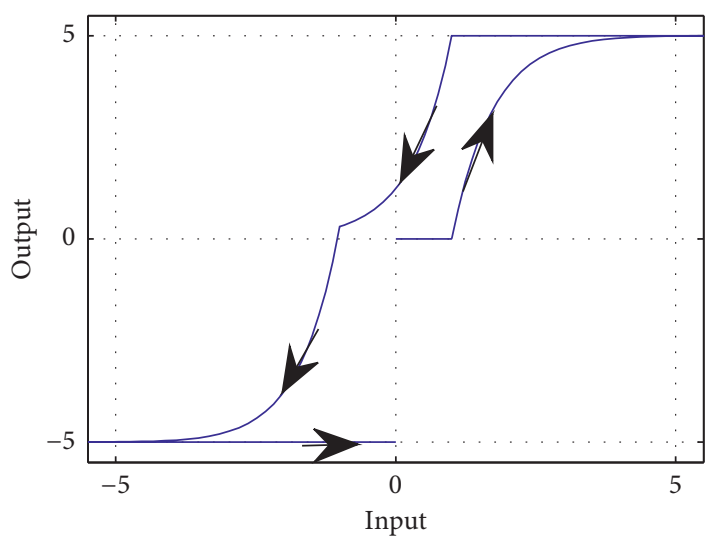

(a)

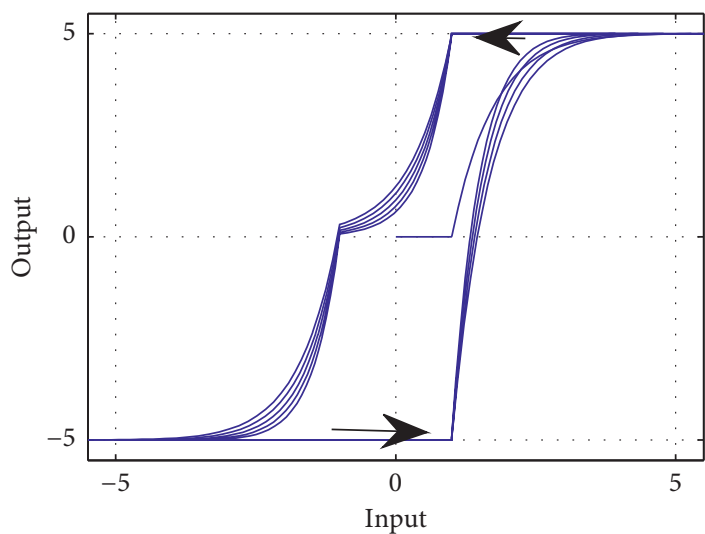

(c)

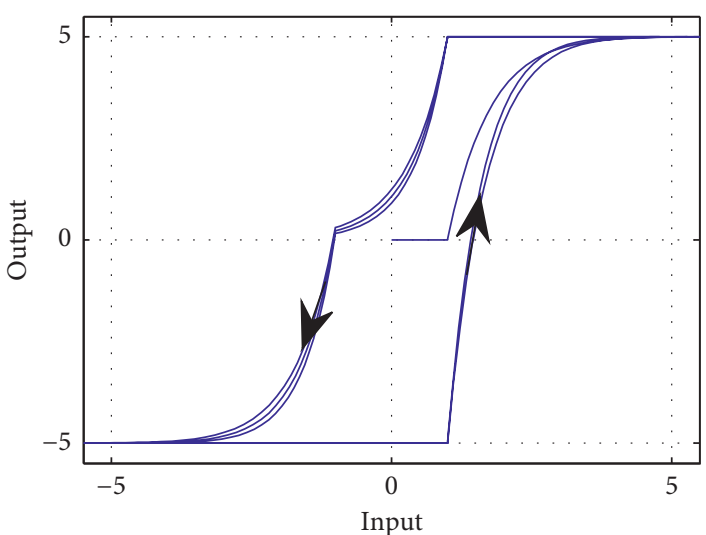

(b)

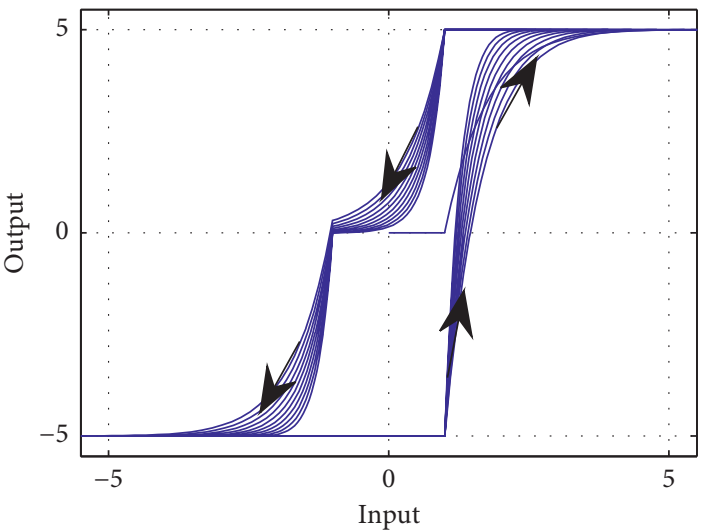

(d)

Figure 9: Time evolution of the convex combination hysteretic behaviour. (a) Time: $1 \mathrm{~s}$. (b) Time: $3 \mathrm{~s}$. (c) Time: $5 \mathrm{~s}$. (d) Time: $10 \mathrm{~s}$.

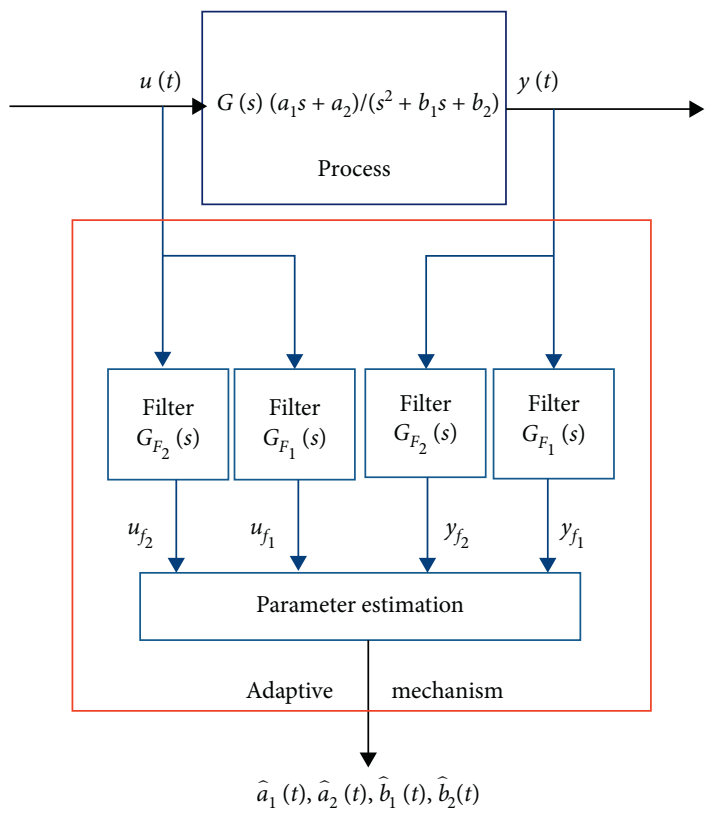

FIGURE 10: Parameter estimation with the corresponding filtered signals. 


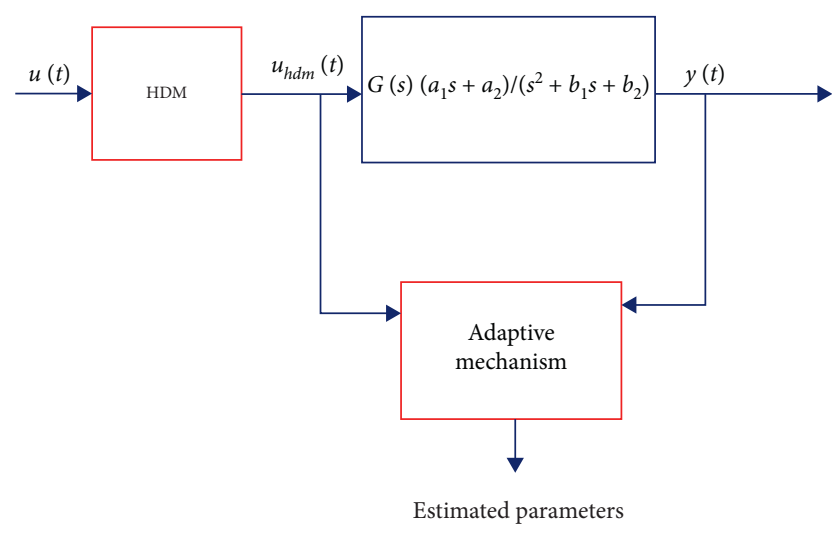

FIgURe 11: Parameter estimation block diagram with the HDM scheme in it.

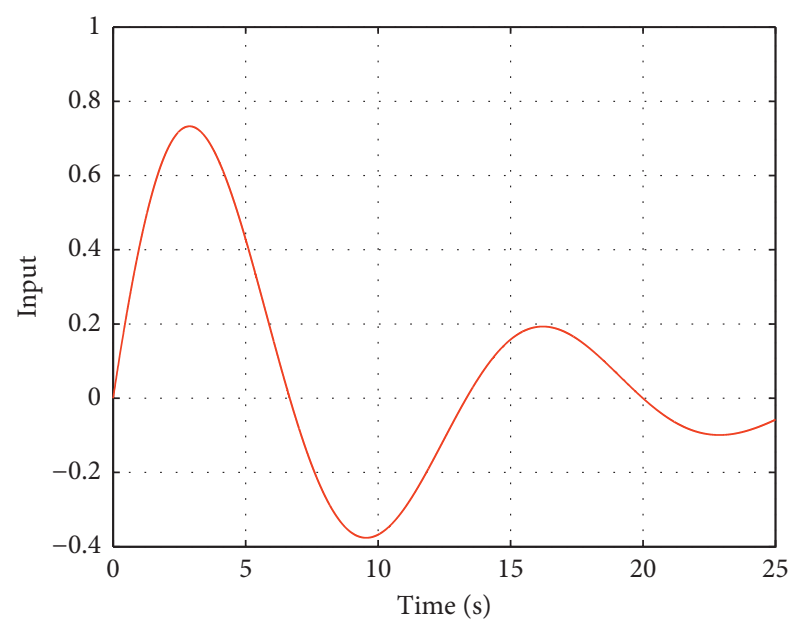

FIgURE 12: Input signal for numerical experiments.

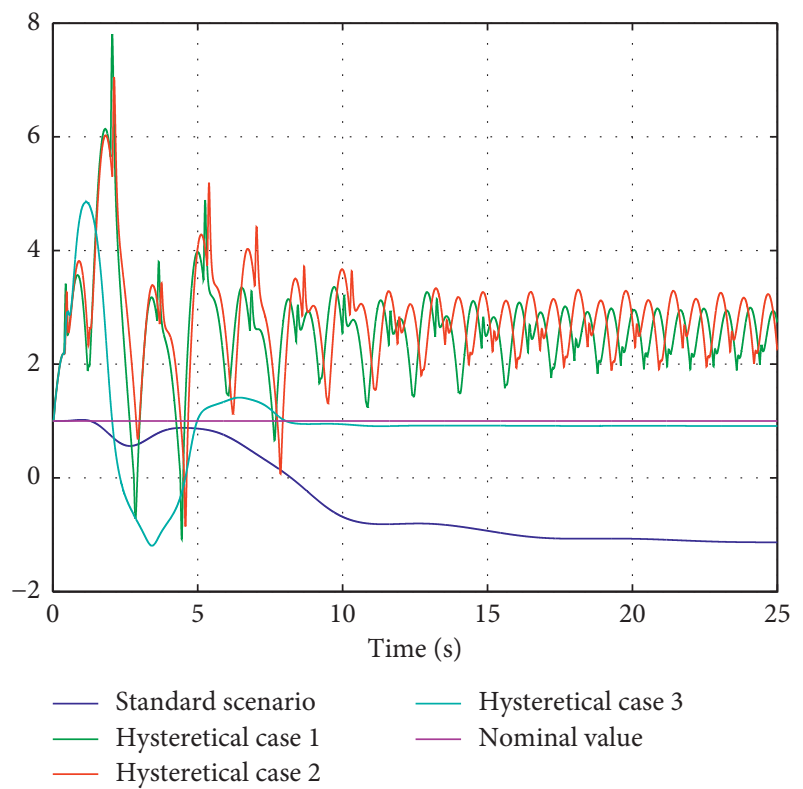

FIgURE 13: Parameter estimation signals $\widehat{a}_{1}(t)$ for each studied scenario.

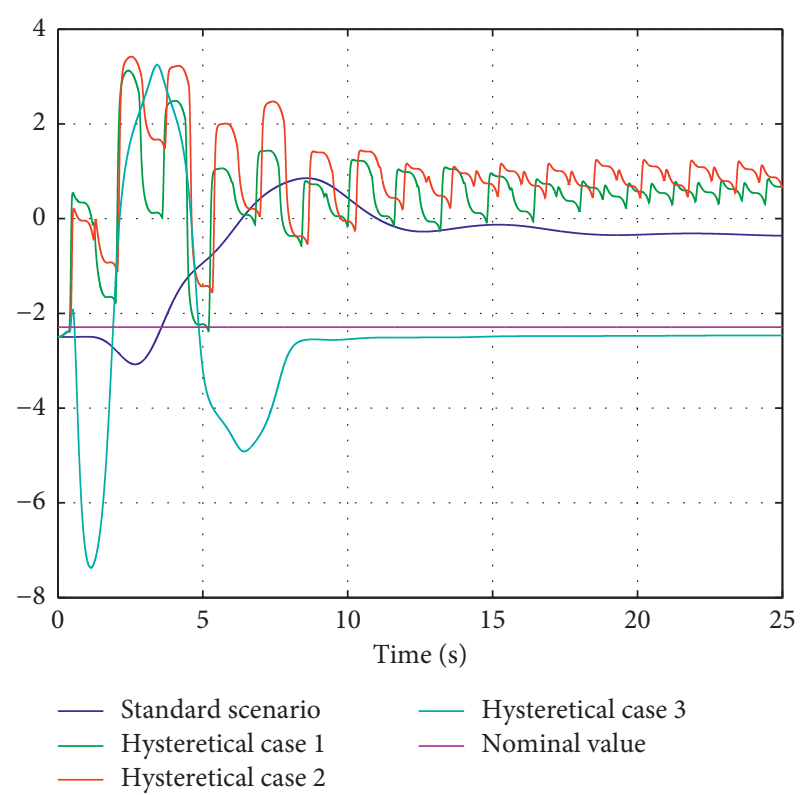

Figure 14: Parameter estimation signals $\widehat{a}_{2}(t)$ for each studied scenario.

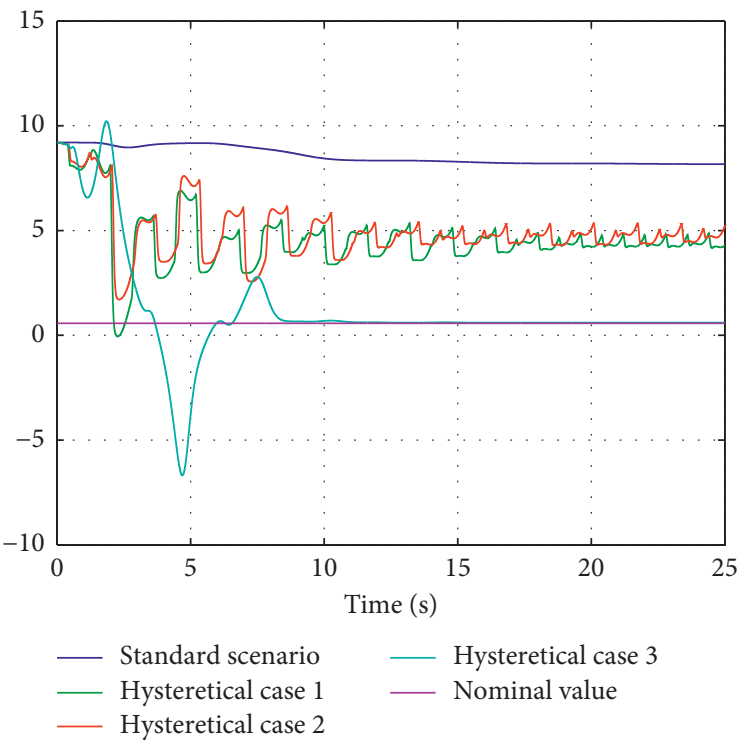

Figure 15: Parameter estimation signals $\widehat{b}_{1}(t)$ for each studied scenario.

where $e(t)=\hat{\theta}(t) \varphi(t)-y(t)$ and $\gamma$ is a given positive constant called the adaptation gain. With the above, the precise parameter estimation system results in the following:

$$
\begin{aligned}
& \dot{\hat{a}}_{1}=-\gamma u_{f 2}\left[\widehat{a}_{1} u_{f_{2}}+\widehat{a}_{2} u_{f_{1}}+\widehat{B}_{1} y_{f_{2}}+\widehat{B}_{2} y_{f_{2}}-y\right], \\
& \dot{\hat{a}}_{2}=-\gamma u_{f_{1}}\left[\widehat{a}_{1} u_{f_{2}}+\widehat{a}_{2} u_{f_{1}}+\widehat{B}_{1} y_{f_{2}}+\widehat{B}_{2} y_{f_{2}}-y\right], \\
& \dot{\widehat{B}}_{1}=-\gamma y_{f_{2}}\left[\widehat{a}_{1} u_{f_{2}}+\widehat{a}_{2} u_{f_{1}}+\widehat{B}_{1} y_{f_{2}}+\widehat{B}_{2} y_{f_{2}}-y\right], \\
& \dot{\widehat{B}}_{2}=-\gamma y_{f_{1}}\left[\widehat{a}_{1} u_{f_{2}}+\widehat{a}_{2} u_{f_{1}}+\widehat{B}_{1} y_{f_{2}}+\widehat{B}_{2} y_{f_{2}}-y\right] .
\end{aligned}
$$




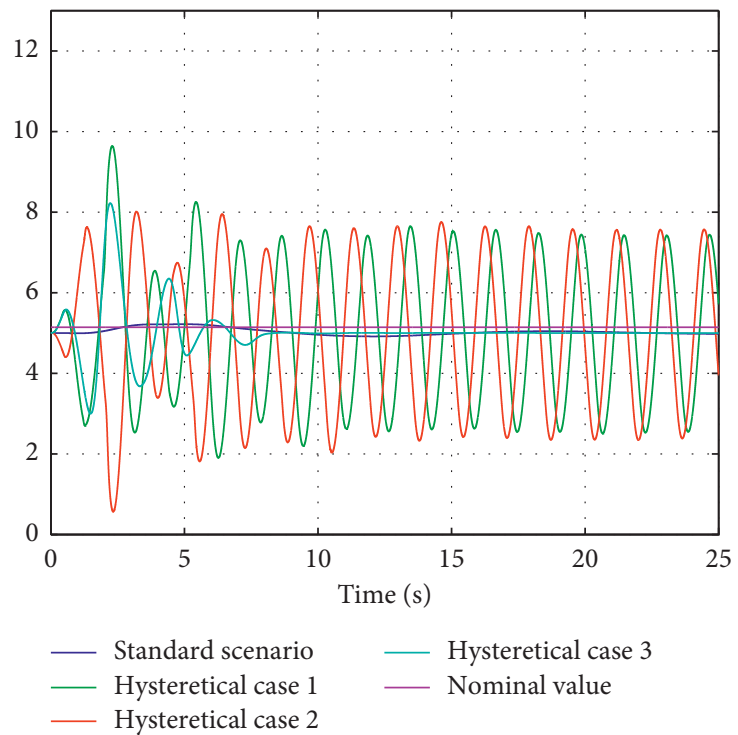

Figure 16: Parameter estimation signals $\widehat{b}_{2}(t)$ for each studied scenario.

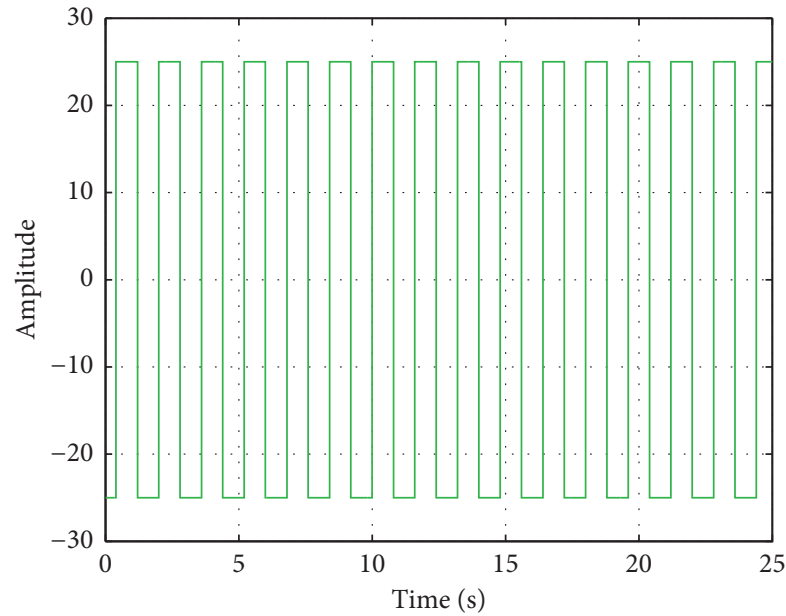

(a)

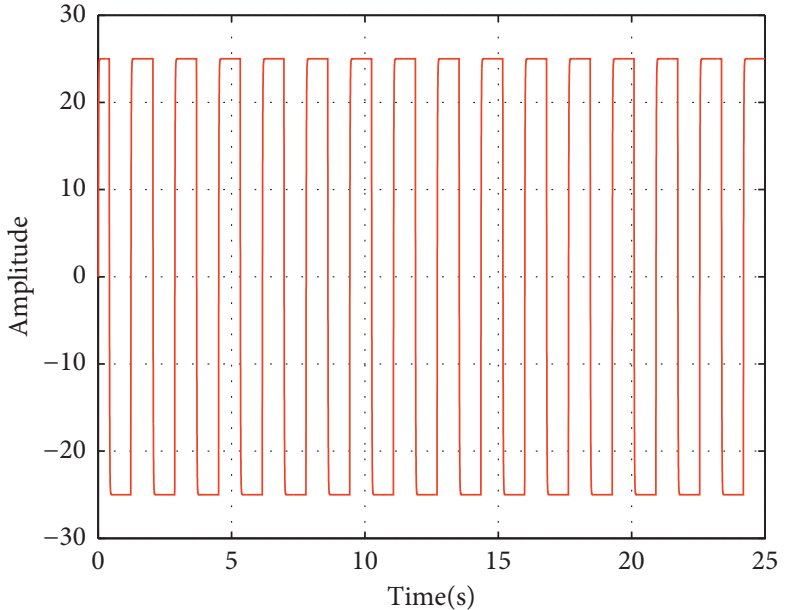

(b)

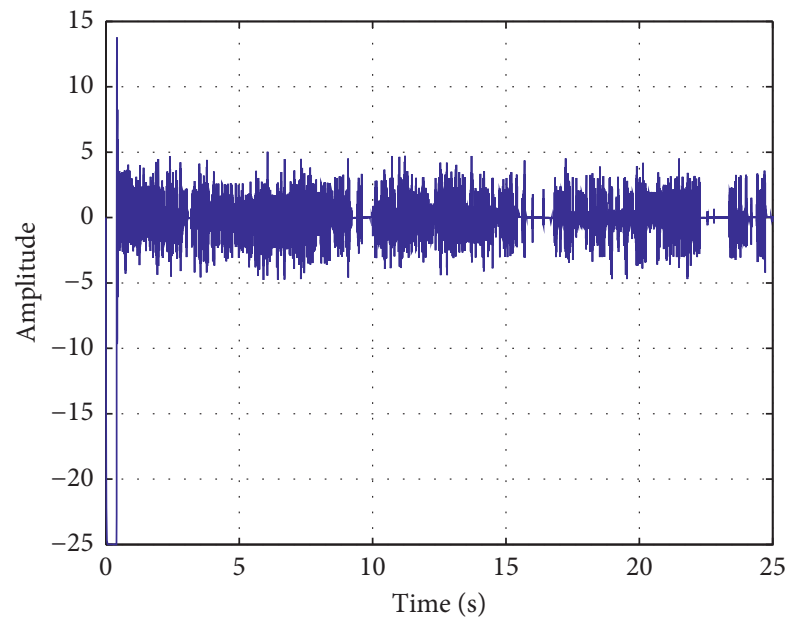

(c)

FIGURE 17: Modulated signals from the HDM block with three different hysteretic models. 


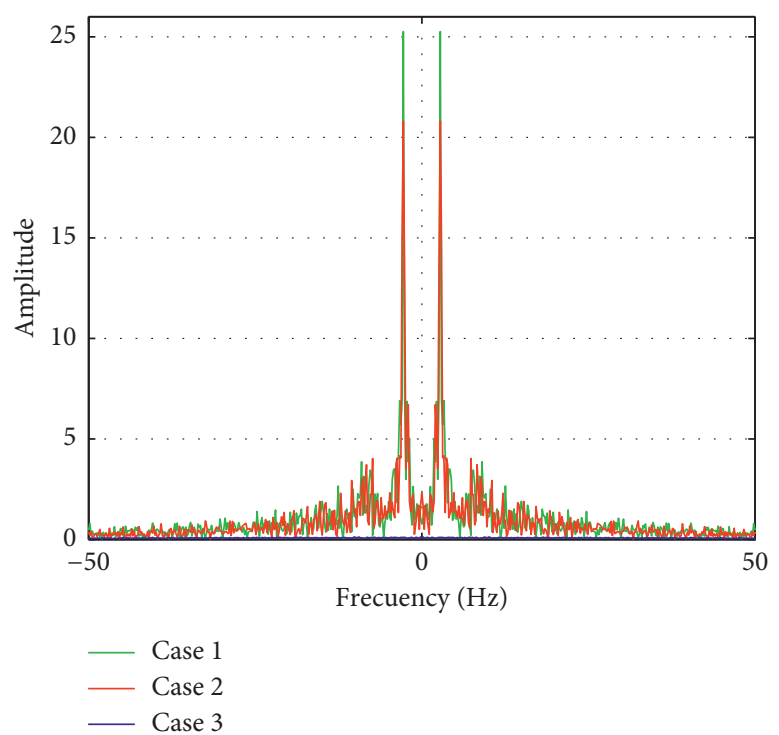

(a)

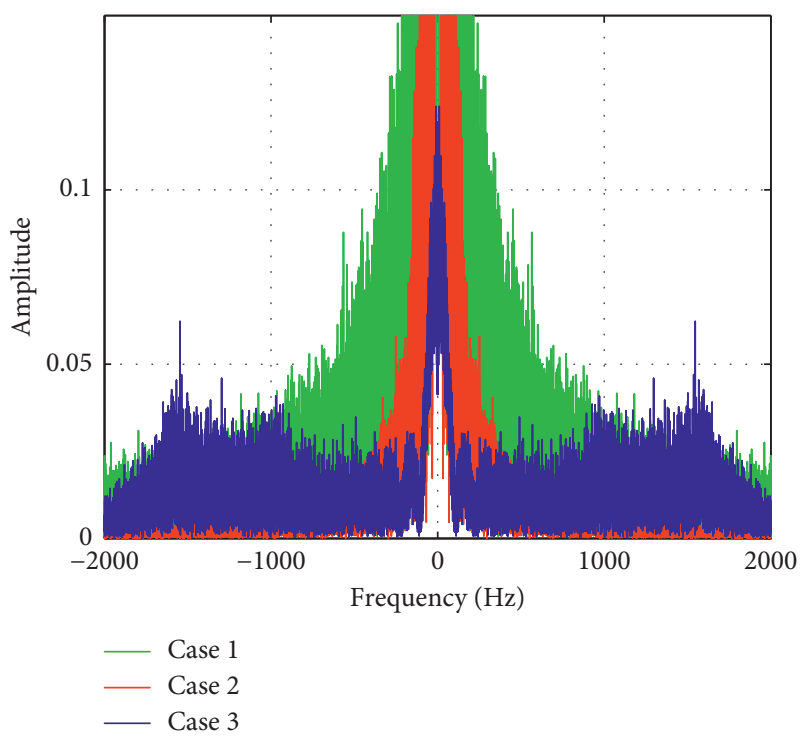

(b)

FIGURE 18: Frequency analysis of modulated signals is shown in Figure 17.

\section{Numerical Experiments and Results}

This section presents numerical experiments that evidence the effectiveness of the proposed HDM scheme in improving the estimation parameter performance. Four different parametric estimation scenarios are numerically implemented and analysed to carry out the comparison study. First scenario corresponds to the standard Gradient Algorithm, that is, a parameter estimation method without using the HDM block in it, see Figure 10. The other remaining three scenarios use the improved parametric estimation method by using the HDM block as it is shown in Figure 11. Each scenario is implemented with the hysteretic model cases commented in the previous section. Therefore, given system (5) as the process, the parametric estimation (11) is done with selected values $\gamma=100, \lambda_{1}=10$, and $\lambda_{2}=5$ and initial conditions $\widehat{a}_{1}(0)=1, \widehat{a}_{2}(0)=-2.5, \widehat{B}_{1}(0)=0.8$, and $\widehat{B}_{2}(0)=5$. Besides, for the scenarios that use the HDM block, see Figure 1, the following values are programmed: for the hysteretic Case 1, switch on/off $=\{10,-10\}$ and output on/ off $=\{25,-25\}$; for the hysteretic Cases 2 and 3, models (1) and (2), respectively, values are set as $a=10, b=25$, and $\alpha=100$.

The main objective of these experiments is to numerically evidence which method exposes the best parameter estimation performance. Hence, the precision of parameter estimation is observed and commented for each method. In the numerical experiments, the signal illustrated in Figure 12 is programmed as the excitation input signal to the system shown in Figures 10 and 11. This signal was specially conceived to resemble a classical control signal with overshooting and converging to zero as time goes on. It is then noted that this signal is not a persistent excitation signal on the whole time horizon. Therefore, this scenario represents an important challenge to any parameter estimation algorithm.
First, Figure 13 shows the numerical experiment results for each method related to the parameter estimation signal $\widehat{a}_{1}(t)$. Clearly, Case 3 , the case with the new hysteresis model, does the best estimation of the parameter $\widehat{a}_{1}$ since it converges to the nominal value of the original plant. The same conclusion is also observed in Figures 14 and 15 for the parameter estimation related to $\widehat{a}_{2}(t)$ and $\$$ $\widehat{b}_{1}(t)$, respectively. On the contrary, note that, in Figure 16, the standard estimation does an acceptable estimation; however, the case with the proposed hysteresis model also has a good performance for the estimation of parameter $\widehat{b}_{2}(t)$.

In summary, from the previous numerical evidences, it can be inferred that the parameter estimation method based on the HDM scheme by using the novel hysteresis model presents the best performance. To roughly justify why our method works better, Fourier analysis is studied and described as follows. Recalling the basic Fourier theory, any periodic function can be reproduced as a summation of a constant and infinite sine and cosine time functions called harmonics. Then, it can be inferred that a kind of persistent excitation signal requires the frequency content to be uniformly distributed. Hence, the Fourier analysis is presented for the corresponding output signal coming from the HDM block for Cases 1-3. These output signals are shown in Figures 17(a)-17(c), respectively. Moreover, the Fourier analysis results are depicted in Figure 18. From these graphics, it is clearly evident that the processed signals corresponding to Cases 1 and 2 have a high-energy harmonic. This provokes nonhomogeneous energy distribution on the frequency content, and as a consequence, it reduces the performance of the corresponding parameter estimation process. While the case with the proposed hysteresis model has a better harmonic distribution, it is useful to improve the performance of the estimation process. 


\section{Conclusions}

This paper exposes two main novelties that are worth emphasizing. Firstly, it proposed a mathematical model that captures the nature of a hysteresis phenomenon. Furthermore, the characteristics provided by this model may be favourable for different engineering applications. For instance, here, it was presented that the recent hysteresis model programmed into the Hysteresis Modulator scheme is adequately applied to adaptive scenarios, which is the second contribution of this paper. Here, it was demonstrated through numerical experiments that the modulator with hysteresis within a parametric estimation scheme produces the best result on estimating the system parameters. However, its performance depends on properly selecting the hysteresis parameters. Hence, as a future work, it is expected to propose an algorithm that appropriately selects the hysteresis parameters. In conclusion, this paper has presented a novel hysteresis dynamic model, and it was adequate to be implemented in a remodelled signal modulator. Hence, a new Hysteresis Delta Modulator was obtained and successfully applied to improve the performance of the system based on parameter estimation process.

\section{Data Availability}

All data and models generated or used during the study are included within this article.

\section{Conflicts of Interest}

The authors declare that they have no conflicts of interest regarding the publication of this paper.

\section{Acknowledgments}

This work was partially supported by the Spanish Ministry of Economy and Competitiveness with a Grant no. DPI201564170-R (MINECO/FEDER), Catalonia Government with the research project no. 2014SGR859, and Scholarship for Doctoral Studies Abroad provided by the CONACYT, Mexico.

\section{References}

[1] I. D. Mayergoyz, The Science of Hysteresis: Mathematical Modelling and Applications, Academic Press, Cambridge, MA, USA, 2006.

[2] E. Della, Torre, Magnetic Hysteresis, Wiley, Hoboken, NJ, USA, 2000.

[3] G. Bertotti, Hysteresis in Magnetism: For physicists, Materials Scientists, and Engineers, Academic Press, Cambridge, MA, USA, 1998.

[4] F. Ikhouane and J. Rodellar, Systems with Hysteresis: Analysis, Identification and Control Using the Bouc-Wen Model, John Wiley \& Sons, Hoboken, NJ, USA, 2007.

[5] L. Acho, "Hysteresis modelling and synchronization of a class of RC-OTA hysteretic-jounce-chaotic oscillators," Universal Journal of Applied Mathematics, vol. 1, pp. 82-85, 2013.

[6] A. Visintin, Differential Models of Hysteresis, Springer Science \& Business Media, Berlin, Germany, 2013.
[7] F. Pozo, G. Pujol, and L. Acho, "Vibration control of hysteretic base-isolated structures: an LMI approach," Smart Structures and Systems, vol. 17, no. 2, pp. 195-208, 2016.

[8] A. Malyshev and V. Malyshev, "Optical bistability and hysteresis of a hybrid metal-semiconductor nanodimer," Physical Review B, vol. 84, no. 3, pp. 314-343, 2011.

[9] A. Bergqvist and G. Engdahl, "A stress-dependent magnetic preisach hysteresis model," IEEE Transactions on Magnetics, vol. 27, no. 6, pp. 4796-4798, 1991.

[10] V. Hassani, T. Tjahjowidodo, and T. N. Do, "A survey on hysteresis modeling, identification and control," Mechanical Systems and Signal Processing, vol. 49, no. 1-2, pp. 209-233, 2014.

[11] K. P. Ryu and A. M. Reinhorn, "Real-time control of shake tables for nonlinear hysteretic systems," Structural Control and Health Monitoring, vol. 24, no. 2, 2017.

[12] N. I. P. de León Puig, L. Acho, J. Rodellar, Y. Vidal, and J. E. M. Gutíerrez Arias, "Hysteretic delta modulator to prevent parameter drift in adaptive based controllers," in Proceedings of the 25th Mediterranean Conference on Control and Automation (MED), pp. 48-53, IEEE, Valletta, Malta, July 2017.

[13] C. Tutivén, Y. Vidal, L. Acho, and J. Rodellar, "Hysteresisbased design of dynamic reference trajectories to avoid saturation in controlled wind turbines," Asian Journal of Control, vol. 19, no. 2, pp. 438-449, 2017.

[14] G. Song, J. Zhao, X. Zhou, and J. DeAbreu-Garcia, "Tracking control of a piezoceramic actuator with hysteresis compensation using inverse preisach model," IEEE/ASME Transactions on Mechatronics, vol. 10, no. 2, pp. 198-209, 2005.

[15] L. Acho and Y. Vidal, "Hysteresis modeling of a class of RCOTA hysteretic chaotic generators," in Proceedings of the 5th International Conference on physics and Control, León, Spain, September 2011.

[16] F. Ikhouane, V. Mañosa, and J. Rodellar, "Dynamic properties of the hysteretic Bouc-Wen model," Systems \& Control Letters, vol. 56, no. 3, pp. 197-205, 2007.

[17] F. Ikhouane and J. Rodellar, "A linear controller for hysteretic systems," IEEE Transactions on Automatic Control, vol. 51, no. 2, pp. 340-344, 2006.

[18] C. Natale, F. Velardi, and C. Visone, "Identification and compensation of preisach hysteresis models for magnetostrictive actuators," Physica B: Condensed Matter, vol. 306, no. 1-4, pp. 161-165, 2001.

[19] G.-Y. Gu and L.-M. Zhu, "Motion control of piezoceramic actuators with creep, hysteresis and vibration compensation," Sensors and Actuators A: Physical, vol. 197, pp. 76-87, 2013.

[20] P. Liu, Z. Zhang, and J. Mao, "Modeling and control for giant magnetostrictive actuators with rate-dependent hysteresis," Journal of Applied Mathematics, vol. 2013, Article ID 427213, , 2013.

[21] L. Malesani and P. Tenti, "A novel hysteresis control method for current-controlled voltage-source PWM inverters with constant modulation frequency," IEEE Transactions on Industry Applications, vol. 26, no. 1, pp. 88-92, 1990.

[22] L. Malesani, P. Mattavelli, and P. Tomasin, "High-performance hysteresis modulation technique for active filters," IEEE Transactions on Power Electronics, vol. 12, no. 5, pp. 876-884, 1997.

[23] D. Taylor, "Design of continuously variable slope Delta modulation communication system," Technical Report, Motorola Techincal Document AN1544, 1996.

[24] M. Zapateiro, L. Acho, and Y. Vidal, "An experimental realization of a chaos based secure communication using 
arduino microcontrollers," The Scientific World Journal, vol. 2015, Article ID 123080, , 2015.

[25] M. Zapateiro, Y. Vidal, and L. Acho, "A secure communication scheme based on chaotic duffing oscillators and frequency estimation for the transmission of binary-coded messages," Communications in Nonlinear Science and Numerical Simulation, vol. 19, no. 4, pp. 991-1003, 2014.

[26] B. Peterson, P. Joonsung, K. Nagaraj, and T. Witt, "Sigmadelta modulator for generating a sinusoidal signal," US Patent 9,692,445, 2017.

[27] R. Wang and J. P. Schulte, "High resolution sigma delta modulator for capacitance sensor terminal displacement measurement," US Patent 9,628,104, 2017.

[28] S. Sastry and M. Bodson, Adaptive Control: Stability, Convergence and Robustness, Courier Corporation, Chelmsford, MA, USA, 1989.

[29] C.-C. Fuh and H.-H. Tsai, “Adaptive parameter identification of servo control systems with noise and high-frequency uncertainties," Mechanical Systems and Signal Processing, vol. 21, no. 3, pp. 1437-1451, 2007.

[30] R. L. Boylestad and L. Nashelsky, Electronic Devices and Circuit Theory, Prentice-Hall, Upper Saddle River, NJ, USA, 2012.

[31] J. M. M. Sánchez and J. Rodellar, Adaptive Predictive Control: From the Concepts to plant Optimization, Prentice Hall, Upper Saddle River, NJ, USA, 1995.

[32] K. Ogata and Y. Yang, Modern Control Engineering, PrenticeHall, Upper Saddle River, NJ, USA, 1970.

[33] S. Dai, Z. Ren, and D. S. Bernstein, "Adaptive control of nonminimum-phase systems using shifted laurent series," International Journal of Control, vol. 90, no. 3, pp. 407-427, 2017.

[34] G. Tao, Adaptive Control Design and Analysis, John Wiley \& Sons, Hoboken, NJ, USA, 2003. 